\title{
Powerful processing to three-dimensional facial recognition using triple information
}

\author{
Mohammad Karimi Moridani ${ }^{1}$, Ahad Karimi Moridani ${ }^{2}$, Mahin Gholipour ${ }^{3}$ \\ ${ }^{1,3}$ Department of Biomedical Engineering, Faculty of Health, Tehran Medical Sciences, Islamic Azad University, Iran \\ ${ }^{2}$ Technologies de L'information Collège CDI, Canada
}

\begin{abstract}
Article Info
Article history:

Received Apr 2, 2020

Revised Jun 11, 2020

Accepted Jul 9, 2020

Keywords:

3D image

Facial information

Information processing,

Mean and gaussian curvature

SVM

ABSTRACT

Face Detection plays a crucial role in identifying individuals and criminals in Security, surveillance, and footwork control systems. Face Recognition in the human is superb, and pictures can be easily identified even after years of separation. These abilities also apply to changes in a facial expression such as age, glasses, beard, or little change in the face. This method is based on 150 three-dimensional images using the Bosphorus database of a high range laser scanner in a Bogaziçi University in Turkey. This paper presents powerful processing for face recognition based on a combination of the salient information and features of the face, such as eyes and nose, for the detection of three-dimensional figures identified through analysis of surface curvature. The Trinity of the nose and two eyes were selected for applying principal component analysis algorithm and support vector machine to revealing and classification the difference between face and non-face. The results with different facial expressions and extracted from different angles have indicated the efficiency of our powerful processing.
\end{abstract}

This is an open access article under the CC BY-SA license.

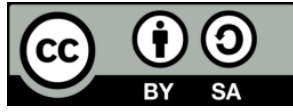

\section{Corresponding Author:}

Mohammad Karimi Moridani,

Department of Biomedical Engineering, Faculty of Health, Tehran Medical Sciences,

Islamic Azad University,

Tehran Province, Tehran, Danesh Rd, Iran.

Email: karimi.m@iautmu.ac.ir

\section{INTRODUCTION}

Face detection has great importance for many as groups. The crucial applications of Face Detection in areas such as human-computer interface, human authentication, criminals face detection, face monitoring systems is undeniable. In all methods proposed to determine the position of specific points in threedimensional images, the nose is regarded as a typical point, and the rest of the points can be located. To determine the location of the nose, several methods were proposed. In some methods, the nearest position of the camera is regarded as the nose, and then the other points are extracted based on the nose position. This assumption is true in the case of no rotation around the $\mathrm{x}$ and $\mathrm{y}$-axis; in fact, the image must be on the front side.

Xu et al. [1] introduced a feature extraction hierarchical plan to identify the positions of the nose tip and nose ridge.

They presented an effective energy idea to show the local distribution of neighboring points and detect the candidate nose tips.

In the end, a support vector machine (SVM) classifier was used to choose the right nose tips.

Dibeklio glu et al. [2,3] displayed strategies for recognizing facial information on 3D facial datasets to empower posture amendment under important posture varieties. 
They presented a statistical strategy to distinguish facial elements using the gradient of the depth map based on preparing a model of local elements. The technique was tried against the FRGC v1 and the Bosphorus databases; however, information with posture varieties was not taken into the investigation.

They likewise presented a nose tip localization and segmentation technique utilizing curvaturebased heuristic investigation. Also, even though the Bosphorus database utilized comprises of 3,396 facial scans, they are acquired from 81 subjects. At last, no correct localization distance error results were displayed.

In other research, Romero-Huertas et al. [4] introduced a graph matching method to determine the places of the nose tip and internal eye corners. They presented the distance to local plane idea to portray the local distribution of neighboring points and identify convex and concave regions of the face. At long last, after the graph matching method has wiped out false landmarks, according to the minimum Mahalanobis distance, the best combination of landmarks points is chosen to the trained landmark diagram model. The technique was tried against FRGC v1 and FRGC v2 databases, including 509 scans and 3271 scans, respectively. They detailed a considerable rate of $90 \%$ with thresholds for the nose tip at $15 \mathrm{~mm}$, and for the internal eye corners at $12 \mathrm{~mm}$.

At last, Perakis et al. [5, 6] displayed techniques for distinguishing important facial points such as eye inner and outer corners, mouth corners, and nose and chin tips) based on 2.5D scans. Neighborhood shape and curvature processing using shape index, expulsion maps, and turn pictures were utilized to find candidate landmark points. These are determined and marked by coordinating them with a statistical facial landmark model $[7,8]$.

The goal of this paper is to find out an efficient face registration method based on triple. Then we will introduce the problems related to the existing systems in face recognition and possible research ways that help to solve these issues. Three dimensional methods could provide better robustness to create diversity than 2D-based methods. Automatic face recognition detection has become one of the most important research topics in image processing due to its application in human-robot interactions, pain detection, lie detection, and other psychological and medical applications.

\section{MATERIAL AND METHOD}

In this method, it is assumed that a true display of a three-dimensional image as a piece of the image for each location $(j, i)$ coordinates $(X, Y, Z)$ the three-dimensional scene. Some of the images received from the device illustrate the data on a form of polygon models, usually called the triangular model. In this case, the range of the image can be detected using the Z-buffer algorithm [1]. As a result, the resulting image may include any number of faces; in that case, it depends on the need and also the imaging device. To avoid the less computational problem, we first seek facial features such as eyes and nose. As a result, this is the first step in image segmentation in related areas of the face features.

Currently, our goal is to distinguish the face triangle on real faces. This method is registered for the position and standard orientation of the face and reduction of the variability of the depth of the face. The triangle distance of the nose and eyes in the image is also calculated.

\subsection{Nose and eyes identification}

The HK segmentation method is used to find the nose and the inner corners of the eyes. For a surface, with a curvature $\mathrm{H}$ mean and $\mathrm{K}$ Gaussian curvature, the facial expression can be identified. In this case, the nose is the maximum point, and the corners of the eyes are the minimum. Based on the average curvature of the face, and because of the leading position and hilly of the nose, it has the highest curvature in the surrounding area, so regarding the face, the points with the maximum curvature are searched. For this purpose, the mean surface curvature is calculated. Afterward, the areas with a greater curvature specified and the area with the greatest curvature will be the tip of the nose. If the curvature of an area is greater, therefore the total amount of curvature in the area will be more obvious. In addition to that, because of the noise, Curvature enhances in one or several pixels, but the high curvature of the searched area is curved, the filter reduces the noise and averages the effects of preventing the wrong location by calculating the mean [9]. The image of the mean curvature, before and after the filtering is shown in Figure 1.

This method determines the position of the nose under any desired angle around the $\mathrm{x}, \mathrm{y}$, and $\mathrm{z}$. The only limitation is more likely if more than half of the nose is hidden around the y-axis. This method utilizes the mean curvature of the face and does not take advantage of the educational and classified data. For this reason, this method has a higher computational speed than the methods that find the nose from different angles. 


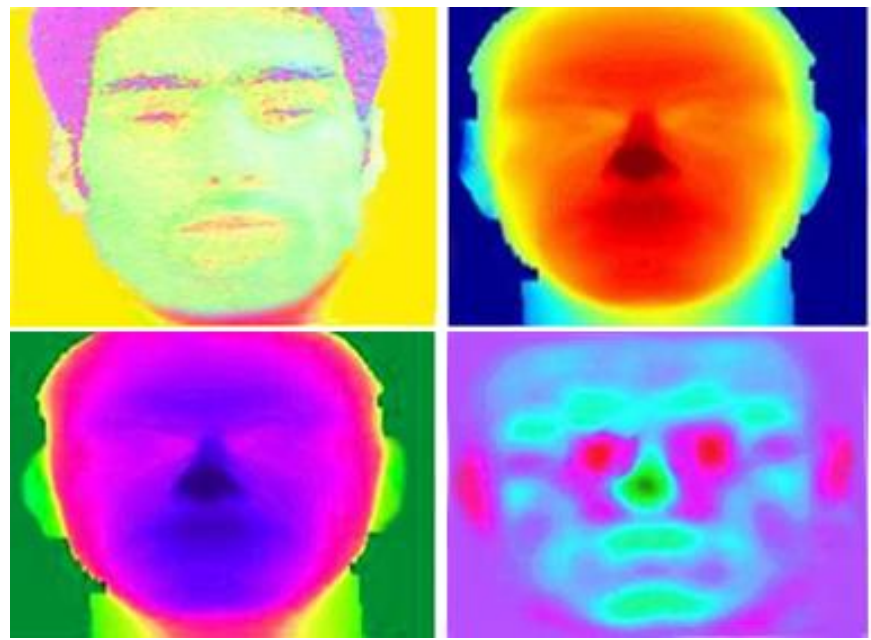

Figure 1. The front part of the face (top right), the image of average curvature (top left), the front part of the face with more light intensity (bottom left), the image of the average curvature after filtering (bottom right)

\subsection{Recognition in image rotation}

The computational cost of the use of the rotating image is high. Initially, a series of areas that are likely to locate the nose and eyes are specified, then to locate the exact area, the rotating image is extracted, and using SVM (Support Vector Machine) classification, the exact located is specified [10, 11]. This method needs primary data and a neural network. To determine the location of the nose, the angles around the y-axis is quantized, and then the images rotate around the y-axis, then the nearest point to the camera is considered as a candidate. After a full rotation around the y-axis, the nose is diagnosed. This method can identify the nose position if the rotation is around the y-axis, and this method is not cost-effective if the rotation happens to the other angles.

\subsection{Three-dimensional face rotation}

Three rotational matrices or their multiplied structure can be used to rotate three-dimensional images around the axes of rotation [12-14]. If we assume that alpha is rotation around axis X, Beta is rotation around axis $\mathrm{Y}$, and Lambda is rotation around the axis of $\mathrm{Z}$, then the formula around each axis will be as (1) to (3). In formula (4) the result is the by multiplying form of the three matrices:

$$
\begin{aligned}
& R_{1}(\alpha)=\left[\begin{array}{ccc}
1 & 0 & 0 \\
0 & \operatorname{Cos} \alpha & -\operatorname{Sin} \alpha \\
0 & \operatorname{Sin} \alpha & \operatorname{Cos} \alpha
\end{array}\right] \\
& R_{2}(\beta)=\left[\begin{array}{ccc}
\operatorname{Cos} \beta & 0 & \operatorname{Sin} \beta \\
0 & 1 & 0 \\
-\operatorname{Sin} \beta & \operatorname{Sin} \alpha & \operatorname{Cos} \beta
\end{array}\right] \\
& R_{3}(\gamma)=\left[\begin{array}{ccc}
\operatorname{Cos} \gamma & -\operatorname{Sin} \gamma & 0 \\
\operatorname{Sin} \gamma & 1 & 0 \\
0 & 0 & 1
\end{array}\right] \\
& R=R_{1} * R_{2} * R_{3}= \\
& {\left[\begin{array}{ccc}
\operatorname{Cos} \beta \cdot \operatorname{Cos} \gamma & -\operatorname{Cos} \beta \cdot \operatorname{Sin} \gamma & \operatorname{Sin} \beta \\
\operatorname{Sin} \alpha \cdot \operatorname{Sin} \beta \cdot \operatorname{Cos} \gamma+\operatorname{Cos} \alpha \cdot \operatorname{Sin} \gamma & -\operatorname{Sin} \alpha \cdot \operatorname{Sin} \beta \cdot \operatorname{Sin} \gamma+\operatorname{Cos} \alpha \cdot \operatorname{Sin} \gamma-\operatorname{Sin} \alpha \cdot \operatorname{Cos} \beta \\
-\operatorname{Cos} \alpha \cdot \operatorname{Sin} \beta \cdot \operatorname{Cos} \gamma+\operatorname{Sin} \alpha \cdot \operatorname{Sin} \gamma & \operatorname{Cos} \alpha \cdot \operatorname{Sin} \beta \cdot \operatorname{Sin} \gamma+\operatorname{Sin} \alpha \cdot \operatorname{Cos} \gamma & \operatorname{Cos} \alpha \cdot \operatorname{Cos} \gamma
\end{array}\right]}
\end{aligned}
$$

As a result, vectors on each spin is obtained with the coordination of the rotated image. The intensity is added to use this matrix, three-dimensional data, and two-dimensional image rotation. So, the image rotation coordination is found. The data matrix is changed as formula (5), and the intensity was added to the last line of image data. 


$$
D=\left[\begin{array}{llllll}
x_{1} & x_{2} & x_{3} & x_{4} & x_{5} & \ldots \\
y_{1} & y_{2} & y_{3} & y_{4} & y_{5} & \ldots \\
z_{1} & z_{2} & z_{3} & z_{4} & z_{5} & \ldots \\
I_{1} & I_{2} & I_{3} & I_{4} & I_{5} & \ldots
\end{array}\right]
$$

The important point here is that two and three-dimensional images of the same size, and both must be accurate with a complete picture of the interaction compliance. The database used; the two-dimensional images are larger than the three-dimensional images. Therefore, first two-dimensional images are resized to be the same size with dimensions of three-dimensional images. After that, a common point in both images is marked, and two images are characterized by $\mathrm{y}$ and $\mathrm{x}$ overlap, then the data is available for each coordination $[15,16]$. Due to the change of the data matrix, a rotation matrix also needs to be changed. Since the intensity data does not affect the pixel location, the rotation matrix will be as formula (6).

Where the available zero and one data are added to the rotation matrix, so the light intensity data is transmitted without altering.

$$
\left[\begin{array}{llll} 
& & & 0 \\
& R & & 0 \\
& & & 0 \\
0 & 0 & 0 & 1
\end{array}\right]
$$

Then the collected data in a two-dimensional matrix are transformed into a two-dimensional rotated image [17].

\subsection{Face detection}

The use of information procedure has several advantages. First of all, the first data is for the depth, not the intensity of the light, so independent of the severity of the light or the light radiation angle or the face size, the obtained image is fixed. Also, these data do not use the reflected light from the face. Therefore, it is not dependent on the skin color changes affected by the makeup or sunlight and sunburn. Generally, the three-dimensional data can be moved and rotated in the desired angle in three-dimensional space; accordingly, the formula is extracted under the desired angle. Therefore, if the image is not at the desired angle, it can be rotated to calculate the image at the desired angle [18-20].

The need for three-dimensional imaging cameras, which are more expensive than two-dimensional imaging cameras, is one of the disadvantages of this method. Facial hair is one of the cases which makes the extraction of depth data difficult [20]. Due to the detection of faces by the trinity of eyes and nose, the system can get along with problems such as hiding the ear, hair, eyebrows, etc. (Figure 2).

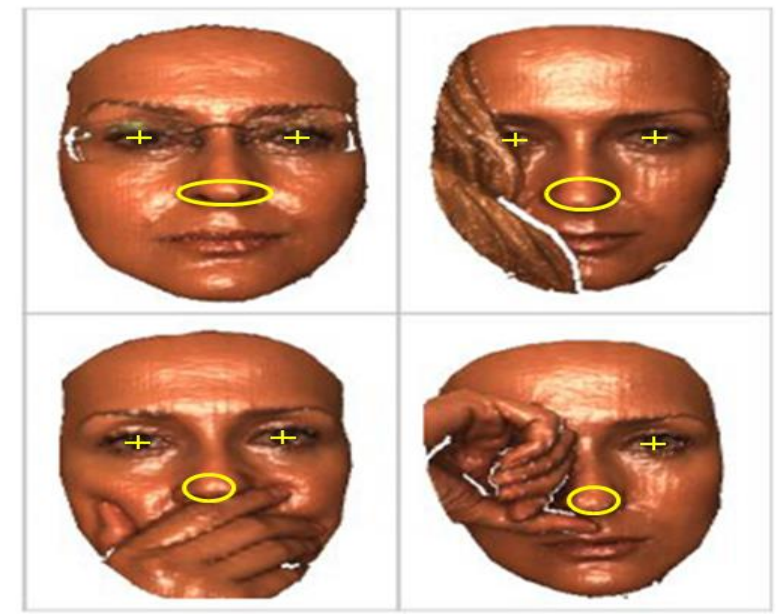

Figure 2. Face detection using nose and eyes

The complete procedure can be extracted through a panoramic camera or multiple photos in different directions. 


\subsection{Experimental testing}

The proposed method implemented on the Bosphorus database on 150 faces shows that this method is $99.7 \%$ accurate in the diagnosis of the position of the nose, $99.3 \%$ correct diagnosis of the position of the eyes, and $96.7 \%$ accurate diagnosis of eye position. In Table 1, the results determine the location of the eyes, nose, and internal parts of the eyes, which were shown in the database.

Table 1. The number of nose and eyes positions which were detected

\begin{tabular}{|c|c|c|c|}
\hline Viewing Angle & Nose Detection & $\begin{array}{l}\text { Left Eye } \\
\text { Detection }\end{array}$ & $\begin{array}{l}\text { Right Eye } \\
\text { Detection }\end{array}$ \\
\hline Front Photo & 143 & 141 & 139 \\
\hline Rotate 25 degrees around $\mathrm{Y}$ & 143 & 143 & 139 \\
\hline $\begin{array}{l}\text { Low rotation } \\
\text { around the } \mathrm{z} \text {-axis }\end{array}$ & 143 & 143 & 141 \\
\hline $\begin{array}{l}\text { High rotation } \\
\text { around the } \mathrm{z} \text {-axis }\end{array}$ & 143 & 143 & 141 \\
\hline Smile Picture & 143 & 142 & 140 \\
\hline Open Mouth & 141 & 141 & 140 \\
\hline The rotation around the $\mathrm{x}$-axis (above) & 143 & 143 & 141 \\
\hline $\begin{array}{l}\text { The rotation around the } \mathrm{x} \text {-axis } \\
\text { (down) }\end{array}$ & 142 & 142 & \\
\hline
\end{tabular}

\section{RESULTS AND DISCUSSION}

The proposed algorithm is implemented on 14 pictures of 150 persons is shown in Table 2 . In Figure 3 the accuracy graph is plotted for different errors.

Table 2. Estimation accuracy of angle for various errors

\begin{tabular}{llll}
\hline The error & Z-Axis & y-Axis & X-Axis \\
\hline $10^{\circ}$ error. & $2198 \%$ & $99.52 \%$ & $98.81 \%$ \\
$6{ }^{\circ}$ Error & $94.88 \%$ & $98.81 \%$ & $92.33 \%$ \\
$3{ }^{\circ}$ Error & $77.46 \%$ & $95.00 \%$ & $67.24 \%$ \\
\hline
\end{tabular}

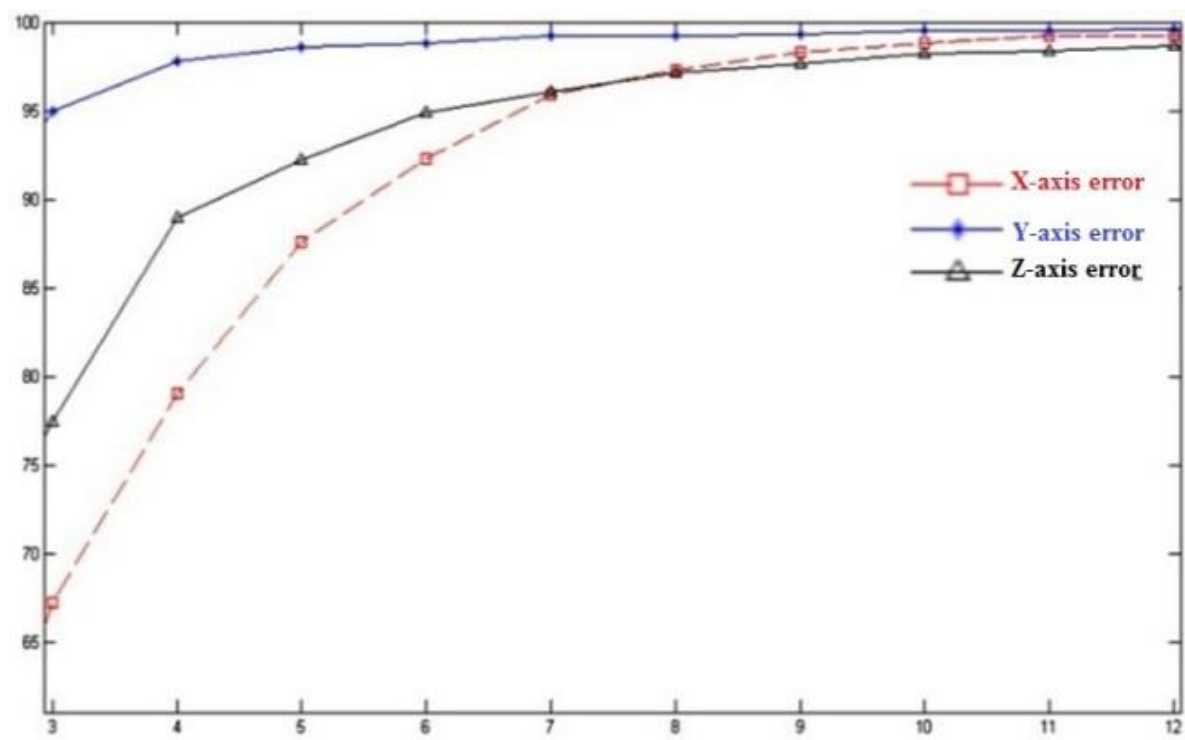

Figure 3. Accuracy diagram for different errors

The running time of this Algorithm, regardless of the time needed to determine the location of the nose, is less than 800 milliseconds. Moreover, this method requires no data for training. Because of the hierarchical structure in case of the wrong estimation of the angles, the wrong estimation of the other angles is also possible. Due to the data mapping of the $\mathrm{Z}=0$, the existence of an error in the calculation of 
the angle in the direction of the z-axis is possible. If we can calculate parameters using the ellipse in threedimensional space, then the accuracy can be increased. Also, if the angle along the $\mathrm{x}$-axis and $\mathrm{y}$ can be determined from the original image along with the angle determination, then the Speed and error of the method will be decreased.

One of the most important capabilities of the human visual system is the ability to recognize the face, which plays an important role in the social life of each person. Our relationship with other people is based on our ability to identify them. One of the most prominent aspects of the human face recognition system is its high accuracy and reliability, but all human beings do face recognition easily and without much effort, while this is not an easy task for computers. The main purpose of this study is to study the effect of selecting the appropriate features of three-dimensional images for face recognition. The results show the success of this method in identifying faces with different characteristics. Different approaches so far are summarized in Table. 3.

Table 3. Comparison of classification accuracy of different methods

\begin{tabular}{|c|c|c|}
\hline Name & 3D face database & $\begin{array}{l}\text { Maximum } \\
\text { Accuracy }\end{array}$ \\
\hline Blanz et al [21] & FRGC & $92 \%$ \\
\hline Dibeklioglu et al [22] & Bosphorus & $79.41 \%$ \\
\hline Mahmood et al [23] & GavabDB & $90 \%$ \\
\hline Berretti et al [24] & UND FRGC v2.0 GavabDB & $82.1 \%$ \\
\hline Perakis et al [25] & UND & - \\
\hline Ding et al [26] & LFW & $92.95 \%$ \\
\hline Proposed method & Bosphorus & $99.52 \%$ \\
\hline
\end{tabular}

Due to the sensitive nature of face detection, high precision is of the utmost importance. Obtained results show that the open mouth increases the possibility of the loss of the nose. Considering that this method is not only to identify the nose and eyes identification are also considered, so this weakness is covered, but in the situation in which both eyes are covered or somehow that part of the image is lost or if the mouth is open; the identification will be less than when the mouth is closed.

\section{CONCLUSION}

Human face recognition by machine through $3 \mathrm{D}$ images has become an active research phase in image processing communities, pattern recognition, neural networks, and computer vision.

The face plays an essential role in identifying people and showing their feelings at the community level. The human ability to recognize faces is remarkable. We can recognize thousands of memorable faces in our lifetime and, at a glance, recognize familiar faces even after years of separation. Face recognition has become an important issue in applications such as security systems, credit card control and crime identification.

In this article, a new method to face recognition automatically using 3D face images was presented. The geometric features of the face were used to identify faces with the help of neural networks. The results showed. The results of using the proposed method in this paper with the help of Bosphorus database showed the highest percentage of accuracy compared to previous methods.

\section{ACKNOWLEDGMENT}

I would like to express my deepest appreciation to Professor Bulent Esnikor from Boğaziçi University for providing me with significant data that greatly assisted the research.

\section{REFERENCES}

[1] Xu C., Tan T., and Wang Y., Quan L., "Combining local features for robust nose location in 3D facial data," Pattern Recognition Letters, vol. 27, no. 13, pp. 62-73, 2006.

[2] Dibeklioglu H., Part-based 3D face recognition under pose and expression variations, Master's thesis, Bo_gazi_ci University, 2008.

[3] Dibeklioglu H., Salah A., Akarun L., "3D facial landmarking under expression, pose, and occlusion variations," In Proc. 2nd IEEE International Conference on Biometrics: Theory, Applications and Systems, pp. 1- 6, 2008.

[4] Romero-Huertas M., Pears N., "3D facial landmark localization by matching simple descriptors," In Proc. 2nd IEEE International Conference on Biometrics: Theory, Applications and Systems, 2008. 
[5] Perakis P., Passalis G., Theoharis T., Toderici G., Kakadiaris I., "Partial matching of interpose 3D facial data for face recognition," In Proc. 3rd IEEE International Conference on Biometrics:Theory, Applications and Systems, pp. 439-446, 2009.

[6] Perakis P., Theoharis T., Passalis G., Kakadiaris I., "Automatic 3D facial region retrieval from multi-pose facial datasets, In Proc. Eurographics Workshop on 3D Object Retrieval, pp. 37-44, 2009.

[7] Xie W, Shen L, Yang M, Lai Z., "Active AU based patch weighting for facial expression recognition," Sensors (Basel), vol. 17,pp. 275, 2017.

[8] S. Berretti, A. Del Bimbo, P. Pala, "Sparse matching of salient facial curves for recognition of 3D faces with missing parts," IEEE Transactions on Information Forensics and Security, vol. 8, no. 2, pp.374-389, 2013.

[9] Andrew J.Logan, Gael E.Gordon, GunterLoffler, "Contributions of individual face features to face discrimination," Vision Research, vol. 137, pp. 29-39, 2017.

[10] S. Ravi, S. Wilson, "Face detection with facial features and gender classification based on support vector machine," 2010 IEEE International Conference on Computational Intelligence and computing Research, ISBN: 9788183713627,2010

[11] T. Verma and R. K. Sahu, "PCA-LDA based face recognition system \& results comparison by various classification techniques," 2013 International Conference on Green High Performance Computing (ICGHPC), Nagercoil, pp. 1-7, 2013.

[12] Zhou, S., Xiao, S. “3D face recognition: a survey.” Hum. Cent. Comput. Inf. Sci. vol. 8, pp. 35, 2018.

[13] Borade S.N., Deshmukh R.R., Shrishrimal P., "Effect of distance measures on the performance of face recognition using principal component analysis," In: Berretti S., Thampi S., Srivastava P. (eds) "Intelligent systems technologies and applications," Advances in Intelligent Systems and Computing, vol. 384, 2016.

[14] S.M.S. Islam, R. Davies, M. Bennamoun, R. "Owens and Ajmal Mian, "Multibiometric human recognition using 3D ear and face features," Pattern Recognition, vol. 46, no. 3, pp. 613-627, 2013.

[15] X.Zhao, W.Zhang, G.Evangelopoulos, D.Huang, S.Shah, Y.Wang, I.Kakadiaris and L. Chen, "Benchmarking asymmetric 3D-2D face recognition systems," Proc. 3D Face Biometrics Workshop, in conjuction with IEEE Int'l Conf. on Automatic Face and Gesture Recognition (FG), 2013.

[16] Yueming Wang, Gang Pan, Jianzhuan Liu, "A deformation model to reduce the effect of expressions in 3D face recognition," Visual Computer, vol. 27, no. 5, pp. 333-345, 2011

[17] A. Watt, M. Watt, Advanced Animation, and Rendering Techniques: Theory and Practice, Addison-Wesley, Reading, MA, 1992.

[18] A. Moreno, A. Sanchez, J. Velez, F. Diaz, "Face recognition using 3D surface extracted descriptors," Proceedings of the Irish Machine Vision and Image Processing, 2004.

[19] Naeem Iqbal Ratyal, Imtiaz Ahmad Taj, Muhammad Sajid, Nouman Ali, Anzar Mahmood and Sohail Razzaq, "Three-dimensional face recognition using variance-based registration and subject-specific descriptors," International Journal of Advanced Robotic Systems, pp. 1-16, 2019.

[20] Miller, P., and Lyle, J. The effect of distance measures on the recognition rates of PCA and LDA based facial recognition Tech. rep., Clemson University, 2008.

[21] Blanz V, Scherbaum K, Seidel HP, "Fitting a morphable model to 3D scans of faces," In: Computer vision, pp. 1-8, 2007.

[22] Dibeklioglu H, Salah AA, Akarun L., "3D facial landmarking under expression, pose and occlusion variations," In: Biometrics theory, applications and systems, pp. 1-6, 2008.

[23] Mahmood SA, Ghani RF, Kerim AA., "3D face recognition using pose invariant nose region detector," In: Computer science and electronic engineering conference, 2014.

[24] Berretti S, Del Bimbo PPA., "Sparse matching of salient facial curves for recognition of 3D faces with missing parts," Forensics Secu, vol. 8, pp. 374-389, 2013.

[25] Perakis P, Passalis G, Theoharis T, Toderici G, Kakadiaris IA., "Partial matching of interpose 3D facial data for face recognition," In: Biometrics: theory, applications, and systems, pp. 439-446, 2009.

[26] Hua WG., "Implicit elastic matching with random projections for pose-variant face recognition," In: Comput. Vis. Pattern Recognit, pp. 1502-1509, 2009. 